\title{
蹘Irestice
}

\section{Perfil dos Aplicativos para Auxílio na Doença de Alzheimer Disponíveis para Download}

\author{
Alzheimer's Disease Relief Applications Profile Available for Download
}

\author{
David Nunes de Lima Junior ${ }^{1}$, Gabriela Mie Ejima Basso², Avelino Missialdes Dutra \\ Júnior $^{3}$, Ruan Costa Gonçalves ${ }^{4}$, Ronaldo Ikaro Farias Araujo ${ }^{5}$, Denisse Sales \\ Paula $^{6}$, Renan Soares ${ }^{7}$, André Marinho Paiva Nogueira ${ }^{8}$, Thiago André Gomes Costa \\ Pereira $^{9}$, José Lopes Tabatinga Neto ${ }^{10}$, Maria do Socorro de Sousa ${ }^{11}$
}

\begin{abstract}
Resumo
Introdução: A Doença de Alzheimer (DA) representa o tipo mais frequente de demência em idosos. Objetiva-se descrever o perfil dos aplicativos disponíveis para DA e avaliar a preferência dos usuários dentre os perfis. Métodos: Foi feita uma pesquisa na plataforma Google Play Store ${ }^{\mathrm{TM}}$, utilizando o unitermo "Alzheimer", por aplicativos direcionados a pacientes com DA, cuidadores ou profissionais da saúde. Analisou-se as variáveis downloads e avaliações. Foram aplicados testes Chi-quadrado, a fim de verificar a independência entre os dados. Foi feita também análise qualitativa dos principais mecanismos destacados em aplicativos. Resultados: Em relação à população geral de aplicativos, os que contêm exercícios para memória são significativamente mais representativos em número de downloads, quando comparado aos que tratam de prevenção. Não houve diferença estatística entre os perfis em avaliações. As principais abordagens dos aplicativos voltados para pacientes foram exercícios para memória, daqueles para cuidadores, ferramentas informativas, e daqueles para profissionais da saúde, mecanismos de auxílio na prática clínica. Conclusão: Aplicativos para auxílio na DA são muito populares, apresentando diferentes abordagens. Estudos posteriores são necessários para analisar a capacidade técnica e a acessibilidade dos portadores de DA e dos cuidadores para utilização dos aplicativos disponíveis.
\end{abstract}

Palavras-Chave: Aplicativos. Doença de Alzheimer. Ferramentas Digitais.

\section{Abstract}

\footnotetext{
1 Graduando. Universidade Federal do Ceará. E-mail: davidn@alu.ufc.br 2 Graduanda. Universidade Federal do Ceará. E-mail: gabiejima@ hotmail.com

3 Especialista. Empresa Brasileira de Serviços Hospitalares. E-mail: avelinodj@uol.com.br

4 Graduando. Universidade Federal do Ceará. E-mail: ruan.costaa@hotmail.com

5 Graduando. Universidade Federal do Ceará. E-mail: r_ikaro@hotmail.com

6 Graduanda. Universidade Federal do Ceará. E-mail: denissesp36@gmail.com

7 Graduando. Universidade Federal do Ceará. E-mail: srenan0905@gmail.com

8 Graduando. Universidade Federal do Ceará. E-mail: andremarinhopn@gmail.com

9 Graduando. Universidade Federal do Ceará. E-mail: thiagogcp23@gmail.com

10 Graduando. Universidade Federal do Ceará. E-mail: zz.neto@hotmail.com

11 Doutora. Universidade Federal do Ceará. E-mail: sousams3@gmail.com

Correspondência: Rua Professor Costa Mendes, 1608 - Bloco Didático, 5a andar, Bairro Rodolfo Teófilo - Fortaleza, Ceará Brasil - CEP: $60.430-140$
}

Rev. Saúde Digital Tec. Educ., Fortaleza, CE, v. 5, n. 1, p.52-65, jan./abr. 2020.

ISSN: 2525-9563 
Introduction: Alzheimer's disease $(A D)$ is the most frequent type of dementia in the elderly. The objective is to describe the profile of the applications available for DA and to evaluate the preference of users among the profiles. Methods: A search was performed on the Google Play Store TM platform, using the term "Alzheimer", for applications directed to patients with $A D$, caregivers or health professionals. The variables downloads and evaluations were analyzed. Chi-square tests were applied in order to verify the independence between the data. A qualitative analysis of the main mechanisms highlighted in applications was also carried out. Results: In relation to the general population of applications, those that contain memory exercises are significantly more representative in number of downloads, when compared to those that deal with prevention. There was no statistical difference between the profiles in evaluations. The main approaches to patientoriented applications were memory exercises, those for caregivers, informational tools, and those for health professionals, assistive mechanisms in clinical practice. Conclusion: Applications to aid in AD are very popular, presenting different approaches. Further studies are needed to analyze the technical capacity and accessibility of patients with $A D$ and caregivers to use the available applications.

Keywords: Applications. Alzheimer's disease. Digital Tools.

\section{Introdução}

A Doença de Alzheimer (DA) é uma doença neurodegenerativa que gera comprometimento cognitivo progressivo, afetando as atividades diárias dos indivíduos acometidos, e representa a forma mais frequente de demência em idosos ${ }^{1}$. As demências, de modo geral, são definidas como uma prioridade de saúde pública global da Organização Mundial da Saúde (OMS) devido ao seu impacto socioeconômico ${ }^{2,3}$. No Brasil, projeções apontam que a prevalência de demências em indivíduos com 65 anos ou mais aumentará, entre 2010 e 2020, de $7,6 \%$ para $7,9 \%$, com incidência de 55.000 novos casos por ano ${ }^{3}$.

Apesar de prevalente, a DA enfrenta desafios no tratamento. Isso se deve, em parte, aos sintomas precoces que, muitas vezes, são sutis e não valorizados pela família. O comprometimento inicial é relacionado frequentemente com comportamentos atribuídos a idade ou a doenças psiquiátricas, como a depressão, dificultando, desse modo, detecção e abordagem precoces ${ }^{4}$.

Nesse sentido, tornam-se necessárias estratégias para melhorar a qualidade de vida dos portadores de DA. Estudos recentes mostram que o uso de aplicativos em aparelhos eletrônicos portáteis, como tablets e smartphones, podem ajudar pessoas com demência a conduzir suas atividades diárias ${ }^{5}$. Considerando que esses aparelhos têm sido mais utilizados que os próprios computadores convencionais, o uso de aplicativos para esses dispositivos representam uma importante fonte de acesso à informação, facilitando diagnóstico e prevenção com abordagem para familiares e para pacientes em potencial6.

Dessa forma, o desenvolvimento de aplicativos que estimulem a interação dos 
portadores de DA vem aumentando consideravelmente. Entretanto, a grande quantidade de aplicativos disponíveis dificulta a escolha do mais adequado para as necessidades individuais dos pacientes, considerando suas atividades diárias e capacidade de autogerenciamento ${ }^{7}$.

Também é importante ressaltar que ser cuidador de um paciente com demência é uma tarefa desgastante e, por isso, está associada a estresse psicológico e esgotamento físico ${ }^{8}$. Intervenções direcionadas aos cuidadores de pacientes com demências visam melhorar seu bem-estar e amenizar a carga emocional provocada pela $\mathrm{DA}^{9}$. Aplicativos em aparelhos eletrônicos voltados para cuidadores já foram criados, entretanto, estudos randomizados ainda são necessários para mostrar a real relevância dos aplicativos para essa população ${ }^{10,11}$.

Face a essas considerações, objetiva-se descrever 0 perfil dos aplicativos disponíveis para DA e avaliar a preferência dos usuários dentre os perfis.

\section{Métodos}

Foi feita uma pesquisa na plataforma Google Play Store ${ }^{\mathrm{TM}}$, entre julho e outubro de 2019, à procura de aplicativos que fossem catalogados para download, utilizando o unitermo "Alzheimer". Os critérios de inclusão de análise foram aplicativos, em qualquer idioma, que preenchessem pelo menos um dos fatores:

1. Fossem direcionados a pacientes com DA.

2. Trouxessem informações que pudessem ajudar os cuidadores dos pacientes.

3. Auxiliassem profissionais de saúde à realização do acompanhamento de seus pacientes portadores de DA.

A partir dos 119 aplicativos selecionados, foram definidos os seguintes perfis:

1. Direcionados para pacientes;

2. Exercícios para memória;

3. Direcionados para cuidadores;

4. Direcionados para profissionais de saúde;

5. Informativos;

6. Prevenção de Alzheimer;

7. Pagos.

A divisão de perfis não constitui uma lista mutuamente exclusiva e, portanto, um aplicativo pode ser categorizado em mais de um perfil.

Para cada um dos aplicativos foram coletadas duas variáveis numéricas: quantidade de downloads e média das avaliações dos usuários (as notas variam de 1 a 5 ).

A quantidade de downloads é disponibilizada na plataforma em um formato numérico discreto, pois assume apenas valores como: 1, 5, 10, 50, 100, 500, 1000 e assim sucessivamente. Dessa forma, para realizar análises 
estatísticas mais confiáveis e alinhadas com o objetivo deste trabalho, optou-se por transformar essa variável em qualitativa ordinal, dividida em duas categorias: i) maior ou igual a 1.000 downloads; ii) menor que 1.000 downloads.

De forma semelhante, transformouse a variável médias das avaliações dos usuários para o tipo qualitativa ordinal, dividida em duas categorias: i) maior ou igual a 4,0; ii) menor que 4,0.

A seguir, foram aplicados testes Chiquadrado para tendência linear (nível de significância $\boldsymbol{\alpha}=0,05)$ a fim de avaliar a independência entre os dados (referentes às duas variáveis ordinais supracitadas) de cada um dos 7 perfis de aplicativos e os dados gerais da população de 119 aplicativos.

Por fim, realizou-se uma análise das funções trazidas em cada aplicativo, bem como destaques que podiam ter associação com a literatura atual sobre a doença.

\section{Resultados}

\subsection{Análise Estatística}

\section{a. Perfil $x$ Quantidade de Downloads}

Os dados apresentados na Tabela 1 evidenciam que há diferenças estatisticamente relevantes na avaliação da quantidade de downloads dentre os sete perfis de aplicativos, quando comparados com a população de 119 aplicativos, mostrando a preferência dos usuários pelo perfil "Exercícios para Memória", enquanto que o perfil "Prevenção de Alzheimer" teve o menor número de downloads.

O perfil de aplicativo que contém Exercícios para Memória teve uma quantidade de downloads estatisticamente superior, quando comparada aos demais perfis de aplicativos, apresentando um Odds Ratio superior aos demais perfis $(\mathrm{P}$-valor $=$ 0,033; Odds Ratio = 2,652).

O perfil de aplicativo que aborda a Prevenção de Alzheimer teve uma quantidade significativamente menor de downloads, com Odds Ratio inferior ao dos demais perfis. ( $P$-valor $=0,008$; Odds Ratio $=0,162$ ).

\section{b. Perfil x Avaliação do Usuário}

Os dados apresentados na Tabela 2 evidenciam que não há diferenças estatisticamente relevantes na comparação entre a amostra geral de 57 aplicativos (representativa da população) que possuem avaliação do usuário e os 7 perfis de aplicativos avaliados.

\subsection{Discussão}

Diversas funcionalidades foram trazidas pelos programadores, caracterizando-se e diferenciando-se pelo direcionamento tomado, em relação ao público-alvo específico. Nesse contexto, 
pode-se dividir as ferramentas das seguintes formas:

\section{a. Aplicativos Direcionados para Pacientes}

Aplicativos direcionados a pacientes apresentam funcionalidades específicas, destacando-se pela variedade de exercícios para memória (item b).

Outras abordagens lançam mão de lembretes diários de tarefas pré-definidas pelos usuários, como o horário de tomar medicamentos.

Um dos aplicativos permite que o cuidador grave mensagem de voz para o portador de Alzheimer com algum recado. Também permite que 0 cuidador selecione arquivos de mídia, como fotos e músicas, para que sejam compartilhados com o paciente em horários definidos, criando vínculo e interação constante entre eles.

Outra aplicação destaca a possibilidade de inserir dados do usuário, como seu endereço e de seu cuidador, de forma a facilitar o retorno à sua residência, caso esteja perdido. Também foi abordado em outra ferramenta digital o uso de "botão de emergência" que encaminharia para o acionamento de serviço de pronto-socorro ou que avisaria ao cuidador caso o paciente saísse do perímetro de segurança pré-definido.

Já outro aplicativo analisado permite ao portador de DA saber suas ações diárias, o que ele precisa fazer, bem como criar sua árvore de relações, para que possa lembrar de alguém. Outra função de grande relevância desta ferramenta digital é a geolocalização, que permite ao indivíduo saber onde ele está, e avisa aos cuidadores caso ele saia do perímetro estabelecido, função importante para quem convive com a demência.

\section{b. Exercícios para Memória}

Os aplicativos encontrados que oferecem exercícios para memória utilizam mecanismos de marcar figuras solicitadas, acertar combinações de elementos, reconhecer números e formas geométricas, resolver quebra-cabeças, analisar a diferença entre objetos, formar palavras, reconhecer cores e padrões, e responder perguntas sobre conhecimentos gerais.

Dois aplicativos que envolvem exercícios de memória, destacam-se por terem uma quantidade expressiva de downloads (mais de 10.000 e mais de 100.000), acompanhada de alta avaliação pelos usuários (4.5 e 4.3). Ambos estão disponíveis em português, fato que, por exemplo, aumenta a acessibilidade à população de países como o Brasil. 0 primeiro aplicativo possui numerosos e diferentes exercícios de estimulação da memória e de outras funções cognitivas. O segundo conta com exercícios de memória e de lógica. Os dois tipos de exercícios possuem associação com efeitos positivos no quadro de pessoas 
com Alzheimer ${ }^{12}$. Em análise, estudos de neuroimagem revelam que indivíduos praticantes de treinamento cognitivo (TC) tiveram efeitos vantajosos de neuroplasticidade, aprimorando a memória de trabalho e sugerindo transferência dos benefícios para outras áreas da vida, o que pode levar à melhora da qualidade de vida ${ }^{13}$. Nesse contexto, pode-se pensar em certa oportunidade de utilizar ferramentas digitais em smartphones como TC. Entretanto, outras análises são necessárias em relação à qualidade oferecida pelos aplicativos.

A frequência do uso das ferramentas de exercício de memória também é outro ponto a ser destacado, visto que o impacto do uso de jogos em smartphones varia com esse fator, como qualquer treinamento realizado em $\mathrm{DA}^{12}$. Entretanto, não se verificou, no atual estudo, aplicativos disponíveis sugerindo frequência e duração de exercícios. Tal fato indica que, mesmo que seja contemplada uma abordagem que traga eficiência similar à TC, a falta de um "guia" de como e quando usá-lo pode impedir que alguns resultados sejam atingidos.

Outro ponto-chave é que estudos que aplicavam TC aliado a outra intervenção tinham maior sucesso do que o treinamento tendo em foco um domínio de restauração cognitiva primária ${ }^{12}$. Portanto, nota-se que o uso de aplicativos sem uma terapia adequada não pode ser promovido para a obtenção de uma melhora do quadro da doença. Seria necessário o esclarecimento para 0 usuário sobre a importância de outros acompanhamentos.

Concomitantemente, a supervisão por um profissional da saúde poderia trazer resultados ainda melhores em relação a situações nas quais esse fator não existe ${ }^{13}$ levando em consideração o uso dos aplicativos disponíveis.

Ao mesmo tempo, aplicativos que não usam base científica em seus testes possivelmente não oferecem tantos benefícios para seus usuários, servindo meramente como um passatempo, sem ter comprovado efeito na qualidade de vida dos portadores. Tal fato, todavia, ainda não parece tão divulgado para os consumidores.

Outra perspectiva a respeito dos benefícios do uso de aplicativos que usem exercícios influenciados por técnicas similares a TC é a análise de que esses podem ser vistos como uma forma de tratamento adjuvante de baixo custo com resultados promissores, caso sejam realizados de forma adequada, o que pode impactar diretamente nos gastos em saúde na sociedade. Em relação a isso, atualmente, a grande maioria dos aplicativos disponíveis para download em auxílio para DA é disponível de forma gratuita.

Dessa forma, aplicativos que trazem exercícios para memória, com foco em portadores de DA estão presentes, em maioria, sem custo e, aliado a isso, são também mais procurados (downloads). 
Tal fato pode ser visto como uma possível busca do cuidador ou do próprio paciente pela melhora da saúde, em um ambiente que seja mais cômodo para eles. Também pode ser questionado se a grande procura por essa modalidade possa ser meramente movida por "algo para passar o tempo", não importando se há base científica na aplicação dos exercícios.

\section{c. Direcionado para Cuidadores}

As variedades de funções para quem acompanha os pacientes foram ainda maiores do que as funções trazidas para os portadores de DA. Destacaram-se aplicativos destinados à informação, socialização e interação entre cuidadores, e aplicativos para alarme.

Sabe-se que ser cuidador de um paciente com DA é um fator de risco para o estresse psicológico e problemas de saúde. Por isso, estratégias que envolvam aceitação e apoio socioemocional trazem benefícios em termos de saúde mental e depressão aos cuidadores ${ }^{8}$.

Os aplicativos destinados à socialização tinham como objetivo oferecer o contato entre cuidadores de idosos por meio de redes sociais e blogs, possibilitando que eles compartilhassem informações sobre sua rotina com o paciente e as dificuldades enfrentadas. Por exemplo, um dos aplicativos analisados oferece interação entre cuidadores por meio das redes sociais e blogs, suporte com mensagens de apoio diárias. Esse também oferece facilidade para contato com vários profissionais de diferentes especialidades como médicos geriatras, fisioterapeutas, terapeutas ocupacionais, psicólogos, enfermeiros, fonoaudiólogos, nutricionistas e advogados por meio de uma rede social. Cabe salientar que também esclarece ao cuidador que essa orientação recebida pela rede social não substitui uma consulta presencial.

Outro aplicativo consiste em uma rede social para cuidadores de pacientes com DA compartilharem informações sobre sua rotina diária com o paciente, o que contribui para a interação e o compartilhamento de informações entre diferentes pacientes e cuidadores, diminuindo assim, os fatores de risco para estresse psicológico e problemas de saúde.

Foram encontrados 61 aplicativos com a finalidade de disponibilizar informações sobre a DA para familiares e cuidadores, mantendo-os esclarecidos sobre a doença. Entretanto, nem todos indicavam as fontes dos dados disponíveis, fornecendo algumas informações de origens duvidosas. Tal fato, em algumas circunstâncias, pode ser um elemento criador de crenças que podem prejudicar o tratamento em DA.

Nesse contexto, cabe lembrar que os cuidadores podem estar passando por momentos difíceis com seus familiares, sendo propensos a aceitarem informações de origem incerta como reais 
numa tentativa desesperada de encontrar uma cura ou uma alternativa que minimize os efeitos da doença, mesmo sem embasamento científico. Por isso, é importante garantir que todos os dados utilizados sejam provenientes de referências confiáveis e que elas sejam explícitas nos aplicativos a fim de evitar discordâncias ${ }^{14}$.

Outro aplicativo simula, com o uso de ferramentas de realidade virtual, o cotidiano e os diferentes sintomas, por meio de três cenários distintos do paciente com Alzheimer. Assim, possibilita ao usuário vivenciar e se colocar no lugar de um portador de DA. Além disso, traz algumas informações básicas sobre a doença.

Embora estudos demonstrem os benefícios do uso dos aplicativos pelos cuidadores, cabe ressaltar que os ensaios realizados apresentaram diversas limitações, como o pequeno tamanho da amostra analisada ou o tempo de análise reduzido. Portanto, ainda que os estudos apresentem resultados positivos, novas pesquisas randomizadas são necessárias para verificar a confiabilidade dos dados obtidos até o momento e mostrar a real influência dos aplicativos para essa população $^{10,11}$.

\section{d. Direcionado para Profissionais da Saúde}

Atualmente, os aplicativos móveis se fazem presentes na prática médica, dado os benefícios que trazem consigo. Dentre as vantagens proporcionadas, observamos a facilidade no gerenciamento de informações e tempo, além da manutenção de um banco de dados relevante de cada paciente, facilitando assim, o seu monitoramento ${ }^{15}$.

Foi observado, também, um aplicativo que acumula informações científicas de revistas de alto impacto, trazendo maior facilidade de acesso para os profissionais de saúde usuários da ferramenta.

Outra abordagem trazida por aplicações é a explanação de exames realizados para rastreio de déficit cognitivo, apresentando instrumentos como Mini Exame do Estado Mental (MEEM) e Escala de Depressão Geriátrica (EDG). Encontrou-se também, um aplicativo utilizado para auxiliar no diagnóstico, avaliação e gestão do paciente, utilizando instrumentos como o Montreal Cognitive Assessment (MOCA) e - Saint Louis University Mental Status (SLUMS).

É importante enfatizar que apesar das vantagens oferecidas, há ainda a necessidade do estabelecimento de padrões de validação para aplicativos móveis médicos, de forma a garantir a qualidade e a segurança dos aplicativos atualmente disponíveis. Tal necessidade advém do principal determinante do valor do aplicativo que é a capacidade de fornecer dados precisos e oportunos, a fim 
de servir ao propósito vital de melhorar o resultado dos pacientes ${ }^{14}$.

\section{e. Acessibilidade}

De acordo com a análise estatística realizada, não se pode afirmar que o preço dos aplicativos tenha sido um fator limitador para obtenção dos mesmos, visto que a amostra de aplicativos pagos é reduzida $(\mathrm{n}=9)$. Apenas um desses aplicativos estava avaliado pelos consumidores. Tal fato também dificulta uma análise da qualidade percebida pelos usuários.

\section{f. Prevenção de Alzheimer}

Cinco aplicativos citam a dieta como ferramenta de prevenção da DA. Entretanto, apesar de existir evidência na literatura, principalmente relacionada à dieta do mediterrâneo, outras formas de dieta não têm ainda comprovação em prevenir o surgimento de demência, como foi citado pelas ferramentas digitais, sobre algumas vitaminas específicas encontradas em frutas cítricas ${ }^{16}$.

Um aplicativo cita fornecer uma ferramenta para cálculo do risco de desenvolver DA. Todavia, não traz referências científicas para comprovação da ferramenta. Outro traz como referência a experiência pessoal de profissionais médicos. Entretanto, tais informações apresentam baixo nível de evidência.

Outros fornecem informações que incentivam treinamento intelectual como forma de diminuição do risco de DA.
Contudo, não se sabe até que ponto as medidas de treinamento intelectual podem ser aplicadas nessas condições, desconsiderando outros fatores de risco.

Foi relatado em estudos que mídias digitais podem ser um meio equivocado ${ }^{14}$. Nesse contexto, torna-se evidente que a disseminação de informações equivocadas sobre a prevenção de DA também pode acontecer em ferramentas em smartphones.

\section{g. Informativos}

Durante a discussão dos resultados, foi identificado que o perfil de aplicativo denominado "Informativos" está distribuído nos perfis abordados nas seções "c" e "f". Dessa forma, todos os aplicativos pertencentes a esse perfil foram debatidos anteriormente.

\section{Conclusão}

Aplicativos que auxiliam na melhora da qualidade de vida de pacientes de DA e seus cuidadores são bastante populares, destacando-se aqueles com exercícios de memória. Aqueles destinados aos profissionais de saúde também estão disponíveis, principalmente para uso informativo e para 0 gerenciamento de dados dos pacientes.

Nota-se, também, que parte dos aplicativos analisados que citavam prevenção da DA, na verdade, não tinham embasamento científico evidente para seus utilizadores. 
Dessa forma, percebe-se que o perfil de aplicativos disponíveis para download tem representação variada. Os aplicativos atuais podem servir como fonte de informação, ferramenta de interação social e apoio a atividades cotidianas, sendo vantajosos e seguros para seus usuários, desde que estejam apoiados em informação científica.

Estudos posteriores são necessários para analisar a capacidade técnica e a acessibilidade dos portadores de DA e dos cuidadores para utilização dos aplicativos disponíveis. Além disso, há necessidade de analisar os efeitos de aplicações com exercícios para memória que não tenham base científica.

A análise do impacto da acessibilidade foi limitada pelo tamanho reduzido da amostra de aplicativos pagos, não permitindo afirmar se o preço é um fator que determina a preferência do usuário.

\section{Agradecimentos}

Agradecimentos a auxílios recebidos pelo CNPq (Conselho Nacional de Desenvolvimento Científico e Tecnológico).

\section{Conflitos de Interesse}

Os autores declaram não haver conflitos de interesse.

\section{Referências}

1. Lane CA, Hardy J, Schott JM, Assadeck $\mathrm{H}$, Toudou Daouda M, Adehossi Omar, et al. Alzheimer's disease. Rev Neurol (Paris) [Internet]. 2018;175(4):261-8. Available from: http://www.ncbi.nlm.nih.gov/pubmed/2887 2215

2. Burlá C, Camarano AA, Kanso S, Fernandes D, Nunes R. Panorama prospectivo das demências no Brasil: Um enfoque demográfico. Cienc e Saude Coletiva. 2013;18(10):2949-56.

3. ORGANIZAÇÃO PAN-AMERICANA DA SAÚDE. Estratégia e plano de ação para a demência em idosos. $54^{\circ}$ Conselho Diretor, 67ª Sessão do Comitê Regional da OMS para as Américas; 28 de setembro a 2 de outubro de 2015; Washington (DC), Estados Unidos. Washington (DC): OPAS; 2015. 4. Manual diagnóstico e estatístico de transtornos mentais [recurso eletrônico]: DSM-5 / [American Psychiatric Association ; tradução: Maria Inês Corrêa Nascimento ... et al.] ; revisão técnica: Aristides Volpato Cordioli ... [et al.]. - 5 . ed. - Dados eletrônicos. - Porto Alegre: Artmed, 2014.

5. Joddrell P, Astell AJ. Studies Involving People With Dementia and Touchscreen Technology: A Literature Review. JMIR Rehabil Assist Technol. 2016;3(2):e10. Available from:

https://www.ncbi.nlm.nih.gov/pubmed/285 82254.

6. Rachel A, Uerj F, Simone M, Alencar DM. de informação e educação em saúde XIX Seminário Nacional de Bibliotecas Universitárias SNBU 2016 Eixo Temático : Ecologia da Informação dispositivos móveis em contexto acadêmico $\mathrm{O}$ uso de aplicativos de saúde para dispositivos móveis como fontes de infor.

7. Anthony Berauk VL, Murugiah MK, Soh YC, Chuan Sheng Y, Wong TW, Ming LC. Mobile Health Applications for Caring of Older People: Review and Comparison. Ther Innov Regul Sci. 2018 May 1;52(3):374-82. Available from: https://www.ncbi.nlm.nih.gov/pubmed/297 14532.

8. Gilhooly KJ, Gilhooly MLM, Sullivan MP, McIntyre A, Wilson L, Harding $E$, et al. A meta-review of stress, coping and interventions in dementia and dementia caregiving. BMC Geriatr [Internet]. 2016;16(1):1-8. Available from: 
http://dx.doi.org/10.1186/s12877-0160280-8

9. Gaugler J, James B, Johnson T, Scholz K, Weuve J. 2016 Alzheimer's disease facts and figures. Alzheimer's Dement [Internet]. 2016;12(4):459-509. Available from:

http://dx.doi.org/10.1016/j.jalz.2016.03.00 1

10. Sikder AT, Yang FC, Schafer R, Dowling GA, Traeger L, Jain FA.

Mentalizing imagery therapy mobile app to enhance the mood of family dementia caregivers: Feasibility and limited efficacy testing. J Med Internet Res. 2019 Mar 1;21(3). Available from:

https://www.ncbi.nlm.nih.gov/pmc/articles/ PMC6715046/pdf/aging_v2i1e12850.pdf 11. Brown EL, Ruggiano N, Page TF, Roberts L, Hristidis V, Whiteman KL, et al. Careheroes web and androidTM apps for dementia caregivers: A feasibility study. Res Gerontol Nurs. 2016;9(4):193203.

12. Kallio EL, Öhman $H$, Kautiainen $H$, Hietanen M, Pitkälä K. Cognitive Training Interventions for Patients with Alzheimer's Disease: A Systematic Review. J

Alzheimer's Dis. 2017;56(4):1349-72.

Available from:

https://www.ncbi.nlm.nih.gov/pubmed/282 22505.

13. Gates NJ, Sachdev P. Is cognitive training an effective treatment for preclinical and early alzheimer's disease? J Alzheimer's Dis. 2014;42:S551-9.

Available from:

https://www.ncbi.nlm.nih.gov/pubmed/251 71716.

14. Wang $Y$, McKee M, Torbica A, Stuckler D. Systematic Literature Review on the Spread of Health-related Misinformation on Social Media. Soc Sci Med [Internet]. 2019;240:112552.

Available from:

http://www.ncbi.nlm.nih.gov/pubmed/3156 1111.
15. Ventola CL. Mobile devices and apps for health care professionals: uses and benefits. P T. 2014;39(5):356-364.

Available from:

https://www.ncbi.nlm.nih.gov/pmc/articles/ PMC4029126/.

16. Gardener S, Gu Y, Rainey-Smith SR, Keogh JB, Clifton PM, Mathieson SL, et al. Adherence to a Mediterranean diet and Alzheimer's disease risk in an Australian population. Transl Psychiatry. 2012;2(June). Available from: https://www.ncbi.nlm.nih.gov/pmc/articles/ PMC3565821/. 
Anexos

Tabela 1: Quantidade de downloads.

\section{QUANTIDADE DE DOWNLOADS}

\begin{tabular}{|c|c|c|c|c|}
\hline PERFIL DO APLICATIVO & Maior ou igual a 1000 & Menor que 1000 & Total & P-Valor \\
\hline Todos os Aplicativos & 44 & 75 & 119 & 1 \\
\hline Direcionados para Pacientes & 25 & 37 & 62 & 0,660 \\
\hline Exercícios para Memória & 14 & 9 & 23 & 0,033 \\
\hline Direcionados para Cuidadores & 19 & 41 & 60 & 0,483 \\
\hline $\begin{array}{l}\text { Direcionados para Profissionais } \\
\text { de Saúde }\end{array}$ & 3 & 8 & 11 & 0,522 \\
\hline Informativos & 15 & 46 & 61 & 0,094 \\
\hline Prevenção de Alzheimer & 2 & 21 & 23 & 0,008 \\
\hline Pagos & 1 & 8 & 9 & 0,117 \\
\hline
\end{tabular}

Fonte: Os Autores.

Rev. Saúde Digital Tec. Educ., Fortaleza, CE, v. 5, n. 1, p.52-65, jan./abr. 2020. ISSN: 2525-9563 
Tabela 2: Avaliação do usuário.

AVALIAÇÃO DO USUÁRIO (NOTA DE 1 A

5)

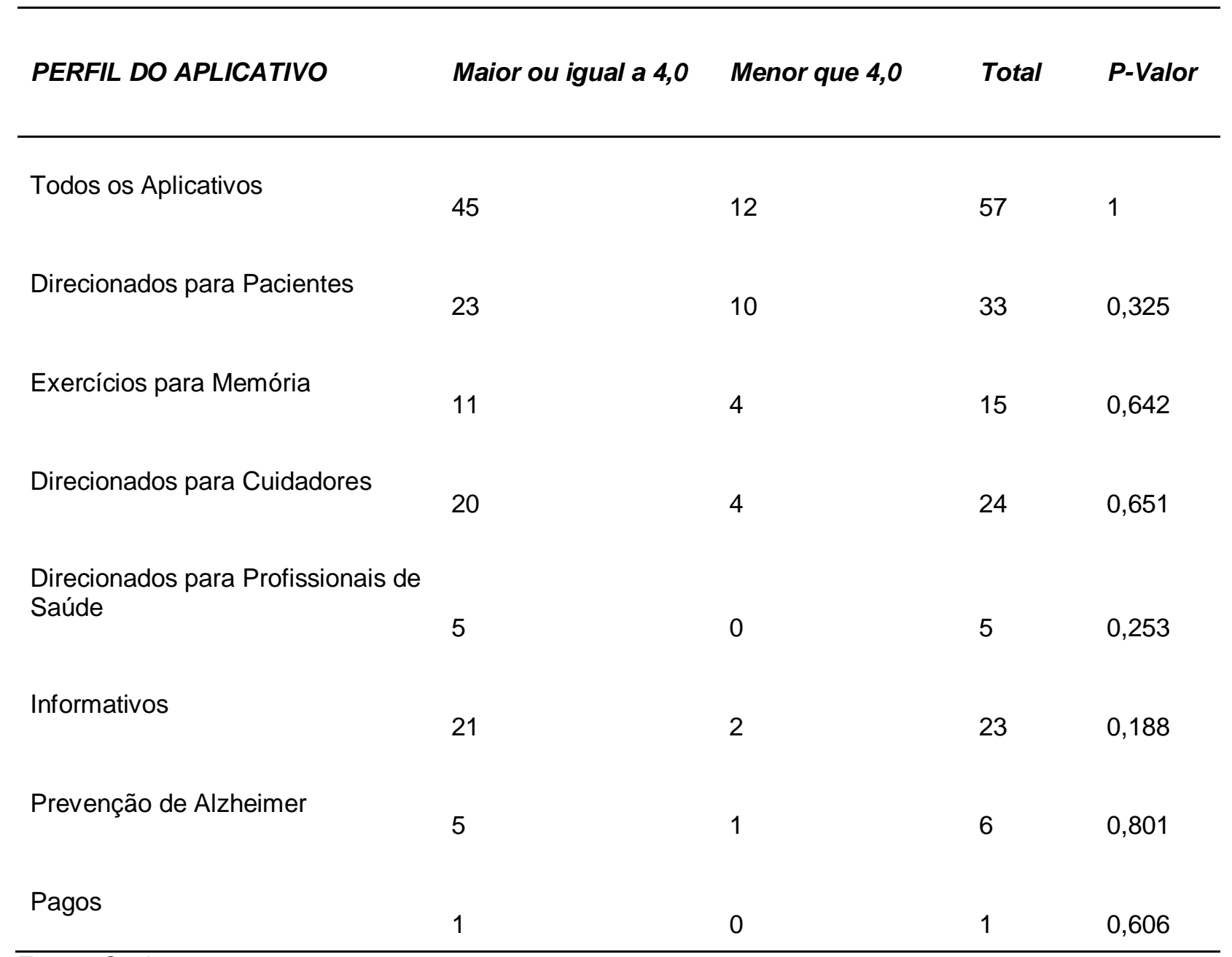

Fonte: Os Autores.

Rev. Saúde Digital Tec. Educ., Fortaleza, CE, v. 5, n. 1, p.52-65, jan./abr. 2020. ISSN: 2525-9563 


\section{Como citar este artigo}

Lima Junior DN de; Basso GME; Dutra Júnior AM; Gonçalves RC; Araujo RIF; Paula DS; Soares R; Nogueira AMP; Pereira TAGC; Tabatinga Neto JL; Sousa MS de. Perfil dos Aplicativos para Auxílio na Doença de Alzheimer Disponíveis para Download. Revista de Saúde Digital e Tecnologias Educacionais. [online], volume 5, n. 1. Editor responsável: Luiz Roberto de Oliveira. Fortaleza, mês e ano, p. 52-65. Disponível em: http://periodicos.ufc.br/resdite/index. Acesso em "dia/mês/ano".

Data de recebimento do artigo: 16/10/2019

Data de aprovação do artigo: 07/02/2020

Data de publicação: 17/04/2020 